\title{
Circular Chromatic Index of Generalized Blanuša Snarks
}

\author{
Mohammad Ghebleh \\ Department of Mathematics \\ Simon Fraser University, British Columbia, Canada \\ mghebleh@math.sfu.ca
}

Submitted: Jul 27, 2007; Accepted: Mar 1, 2008; Published: Mar 12, 2008

Mathematics Subject Classification: 05C15

\begin{abstract}
In his Master's thesis, Ján Mazák proved that the circular chromatic index of the type 1 generalized Blanuša snark $B_{n}^{1}$ equals $3+\frac{2}{n}$. This result provided the first infinite set of values of the circular chromatic index of snarks. In this paper we show the type 2 generalized Blanuša snark $B_{n}^{2}$ has circular chromatic index $3+\frac{1}{1+3 n / 2\rfloor}$. In particular, this proves that all numbers $3+1 / n$ with $n \geqslant 2$ are realized as the circular chromatic index of a snark. For $n=1,2$ our proof is computer-assisted.
\end{abstract}

\section{Introduction}

Let $G$ be a graph and $r>2$. For all $a \in[0, r)$, let $|a|_{r}=\min \{|a|, r-|a|\}$. For $a, b \in[0, r)$, the $r$-circular interval $[a, b]_{r}$ is defined by

$$
[a, b]_{r}= \begin{cases}{[a, b]} & \text { if } a \leqslant b, \\ {[a, r) \cup[0, b]} & \text { if } a>b .\end{cases}
$$

For $a, b \in \mathbb{R},|a|_{r}$ and $[a, b]_{r}$ are defined by first reducing $a$ and $b$ modulo $r$ to $a^{\prime}, b^{\prime} \in$ $[0, r)$. An edge $r$-circular colouring, or an edge $r$-colouring for short, of $G$ is a function $c: E(G) \rightarrow[0, r)$ such that for any two adjacent edges $e$ and $e^{\prime},\left|c(e)-c\left(e^{\prime}\right)\right|_{r} \geqslant 1$. If $G$ admits an edge $r$-colouring, then $G$ is edge $r$-colourable. The circular chromatic index of $G$ is defined by

$$
\chi_{c}^{\prime}(G)=\inf \{r \in \mathbb{R} \mid G \text { is edge } r \text {-colourable }\} .
$$

It is well-known, see [9] for example, that for every finite graph $G$, the infimum in (1) is attained, and that $\chi_{c}^{\prime}(G)$ is rational. It is also known that for every graph $G, \chi^{\prime}(G)=$ $\left\lceil\chi_{c}^{\prime}(G)\right\rceil$, where $\chi^{\prime}(G)$ is the chromatic index of $G$. Hence, by Vizing's theorem, $\Delta(G) \leqslant$ $\chi_{c}^{\prime}(G) \leqslant \Delta(G)+1$. Recall that a graph $G$ is said to be class 2 , if $\chi^{\prime}(G)=\Delta(G)+1$, or 

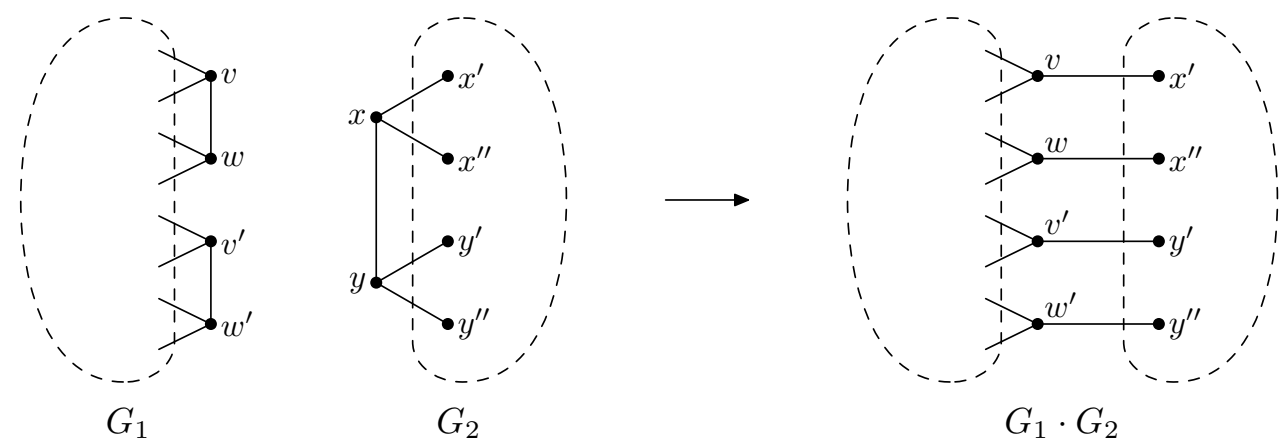

Figure 1: The dot product construction

equivalently, if $\chi_{c}^{\prime}(G)>\Delta(G)$. Afshani et al. [1] proved that if $G$ is a bridgeless cubic graph then

$$
3 \leqslant \chi_{c}^{\prime}(G) \leqslant 11 / 3 .
$$

The upper bound is attained by the Petersen graph. No bridgeless cubic graph other than the Petersen graph with the circular chromatic index greater than $7 / 2$ is known. Kaiser et $a l$. [5] proved that every bridgeless cubic graph with girth at least 14 has circular chromatic index at most $7 / 2$. The circular chromatic index of special classes of graphs has been of interest. For example the circular chromatic index of the flower snarks is studied in [3] and the circular chromatic index of Goldberg snarks and twisted Goldberg snarks is studied in [2]. West and Zhu [8] study the circular chromatic index of Cartesian products of graphs, and toroidal grids in particular.

It is convenient when studying edge-colourings, to allow semiedges in graphs, i.e. edges with only one end-vertex. Having this convention, we may "cut" a given graph into smaller pieces, colour the pieces separately, and then attach the pieces together to obtain a colouring of the given graph. We may also be able to prove that colourings of the pieces are never compatible, thus proving uncolourability of the given graph. For ordinary edge colourings of snarks, such uncolourability arguments usually use the parity lemma. Given an edge 3-colouring of a cubic graph $G$ with order $n$, let $n_{i}$ be the number of semiedges coloured $i$. The parity lemma asserts that

$$
n_{1} \equiv n_{2} \equiv n_{3} \equiv n \bmod 2 .
$$

\section{Generalized Blanuša Snarks}

Having order 18, Blanuša snarks are the smallest snarks after the Petersen graph $P$. They are both obtained by a dot product from two copies of $P$. Given two cubic class 2 graphs $G_{1}$ and $G_{2}$, the dot product $G_{1} \cdot G_{2}$ is constructed by adding four edges to the disjoint union of $G_{1}-\left\{v w, v^{\prime} w^{\prime}\right\}$ and $G_{2}-\{x, y\}$, as shown in Figure 1, where $v w$ and $v^{\prime} w^{\prime}$ are non-adjacent edges in $G_{1}$ and $x$ and $y$ are adjacent vertices in $G_{2}$. This operation was first introduced by Isaacs [4]. 

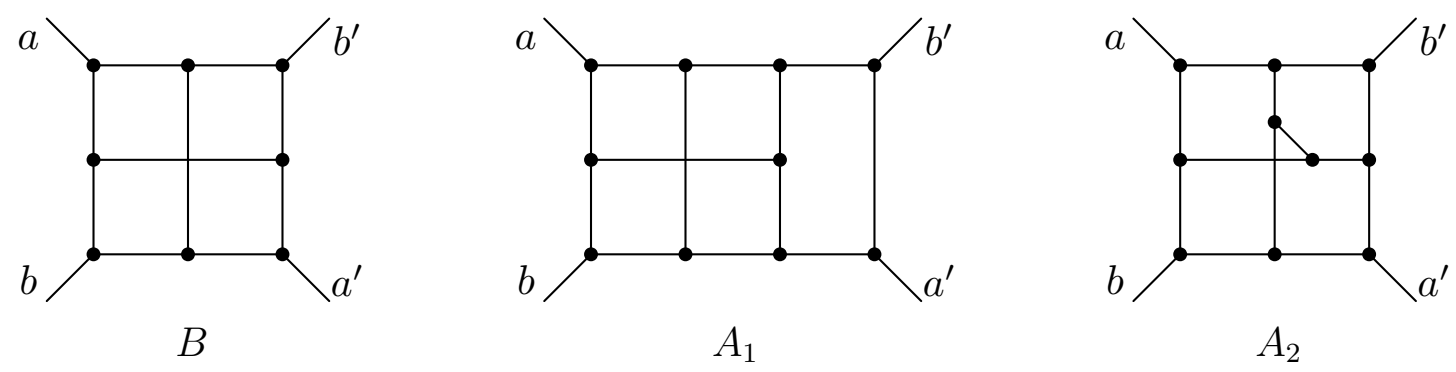

Figure 2: The Blanuša blocks

Note that the dot product $G_{1} \cdot G_{2}$ depends on the choice of the edges $v w, v^{\prime} w^{\prime}$ in $G_{1}$ and the edge $x y$ in $G_{2}$. When $G_{1}=G_{2}=P$, since the Petersen graph is edge-transitive, the choice of $x y$ does not matter. On the other hand, two non-adjacent edges in $P$ can be at distance 2 or 3 in the line graph $L(P)$. The two non-isomorphic snarks obtained by a dot product $P \cdot P$ are called the Blanuša snarks. More precisely, if $v w, v^{\prime} w^{\prime} \in E(P)$ have a common neighbour in $L(P)$, the resulting graph $P \cdot P$ is called the first Blanuša snark, and otherwise, the graph $P \cdot P$ is called the second Blanuša snark.

This construction was generalized by Watkins [7]. We refer to the graph obtained by "cutting" two edges of $P$ which are at distance $i+1$, and keeping the semiedges, as the Blanuša block $A_{i}$. The graph obtained by removing two adjacent vertices of $P$ and keeping the semiedges is called the Blanuša block $B$. These blocks are shown in Figure 2. The labels of the semiedges indicate connections in constructions involving these blocks. The semiedges with label $a$ (resp. b) are always connected to a semiedge with label $a^{\prime}$ (resp. $\left.b^{\prime}\right)$ and vice versa. By this assumption, the first (resp. second) Blanuša snarks is obtained by attaching a copy of $A_{1}$ (resp. $A_{2}$ ) to a copy of $B$. It can be seen in Figure 2 that $A_{1}$ can indeed be decomposed into a copy of $B$ and a single edge. Thus in constructions involving $A_{1}$, we may replace $A_{1}$ by a $B$ and a $K_{2}$ to obtain further decomposition.

Watkins [7] defined two families of generalized Blanuša snarks using the blocks $B$, $A_{1}$, and $A_{2}$. The family $\mathcal{B}^{1}$ consists of the graphs $B_{n}^{1}$ constructed as follows: take $n-1$ copies of the block $B$ and one copy of $A_{1}$, arrange these blocks cyclically, and connect the semiedges $a$ and $b$ of each block to the semiedges $a^{\prime}$ and $b^{\prime}$ of the next block respectively. Note that $B_{1}^{1}$ is the Petersen graph, and $B_{2}^{1}$ is the first Blanuša snark. The family $\mathcal{B}^{2}$ is defined similarly, using the block $A_{2}$ in place of $A_{1}$. Following [7], we refer to members of $\mathcal{B}^{1}$ (resp. $\mathcal{B}^{2}$ ) as type 1 (resp. type 2) generalized Blanuša snarks.

The circular chromatic index of type 1 generalized Blanuša snarks was established by Mazák [6].

Theorem 1. [6] For all $n \geqslant 1, \chi_{c}^{\prime}\left(B_{n}^{1}\right)=3+\frac{2}{3 n}$.

Although it was known that the set $\mathcal{S}=\left\{\chi_{c}^{\prime}(G): G\right.$ is a bridgeless cubic graph $\}$ is infinite, before Mazák's result, only a finite number of values in $\mathcal{S}$ were known. In this paper we prove the following result for type 2 generalized Blanuša snarks which realizes infinitely many new values in $\mathcal{S}$. Note that $B_{1}^{2}$ is the Petersen graph whose circular chromatic index is already known. 
Theorem 2. For all $n \geqslant 1$,

$$
\chi_{c}^{\prime}\left(B_{n+1}^{2}\right)=3+\frac{1}{\lfloor 1+3 n / 2\rfloor}= \begin{cases}3+\frac{2}{3 n+1} & \text { if } n \text { is odd }, \\ 3+\frac{2}{3 n+2} & \text { if } n \text { is even } .\end{cases}
$$

\section{The Upper Bounds}

We first prove the upper bounds of Theorem 2. The structure of the optimum edge colourings of the graphs $B_{n}^{2}$ helps in understanding the proof of the lower bounds, presented in the next section.

Let $G$ be a cubic graph which may contain semiedges. A consecutive colouring of $G$ is any mapping $c: E(G) \rightarrow \mathbb{Z}$ such that for each $v \in V(G)$, if $e, e^{\prime}, e^{\prime \prime}$ are the edges incident with $v$, then the colours $c(e), c\left(e^{\prime}\right), c\left(e^{\prime \prime}\right)$ are three consecutive integers. Obviously, reducing the colours $c(e)$ modulo 3 , one gets a proper edge 3-colouring of $G$. One could also reduce the colours $c(e)$ modulo $3+\varepsilon$ for any given $0<\varepsilon<1$, to obtain an edge $(3+\varepsilon)$-colouring of $G$. The notion of consecutive colouring helps us present circular edge colourings of graphs by integers rather than real numbers.

Lemma 3. Given $n \geqslant 1$, let $\varepsilon=\frac{1}{\lfloor 1+3 n / 2\rfloor}$ and $r=3+\varepsilon$. Then $\chi_{c}^{\prime}\left(B_{n+1}^{2}\right) \leqslant r$.

Proof. We split the proof into two cases depending on the parity of $n$. If $n$ is even, then $\varepsilon=\frac{2}{3 n+2}$. Consider the consecutive colouring of the graph $B$ given in Figure 3(a). Since $3=-\varepsilon$ modulo $r$ and $6=-2 \varepsilon$ modulo $r$, we may combine suitable linear transformations of this colouring for two consecutive copies of the block $B$ in $B_{n+1}^{2}$, to get a colouring $c$ for which $c(a)=c(b)=0$ in the first block, and $c\left(a^{\prime}\right)=c\left(b^{\prime}\right)=3 \varepsilon$ in the second block. This $r$-colouring of two consecutive $B$-blocks is explicitly given in Figure 4 . Since $B_{n+1}^{2}$ contains $n$ copies of the block $B$, combining suitable transformations of these colourings, we get an edge $r$-colouring $c$ of these blocks, for which $c(a)=c(b)=0$ for the first block, and $c\left(a^{\prime}\right)=c\left(b^{\prime}\right)=(n / 2) 3 \varepsilon=1-\varepsilon$ for the last block. On the other hand, since $4=1-\varepsilon$ modulo $r$, the consecutive colouring of $A_{2}$ given in Figure 3(b) can be used to extend $c$ to an edge $r$-circular colouring of $B_{n+1}^{2}$.

If $n$ is odd, then $\varepsilon=\frac{2}{3 n+1}$. Similarly to the previous case, we find a partial edge $r$-colouring $c$ of $B_{n+1}^{2}$ which colours all the edges in all copies of $B$, such that for the block $A_{2}$ we have $c(a)=c(b)=0, c\left(a^{\prime}\right)=1$, and $c\left(b^{\prime}\right)=1-\varepsilon$. This colouring can be extended to $B_{n+1}^{2}$ using the consecutive colouring of $A_{2}$ given in Figure 3(c).

\section{The Lower Bounds}

We need the following two lemmas in our proof of the lower bounds of Theorem 2 . Lemma 4 can be found in [9] in more general settings. Lemma 5 is an easy observation proved in [2]. In the following $\alpha^{\prime}(G)$ denotes the maximum size of a matching in a graph $G$. 


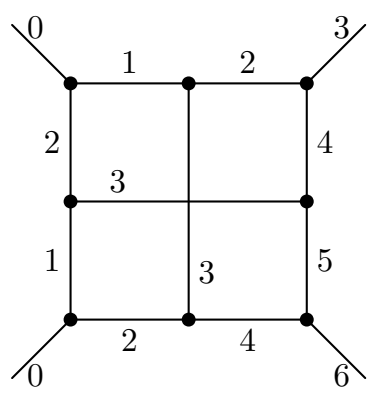

(a)

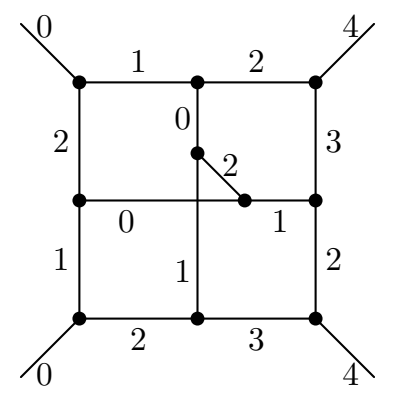

(b)

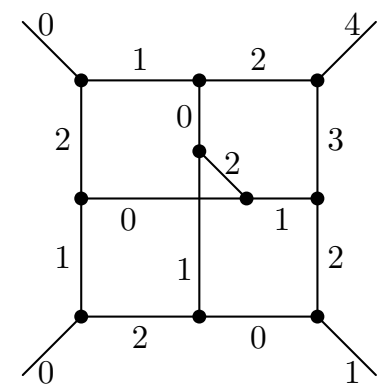

(c)

Figure 3: Consecutive colourings of Blanuša blocks used in Lemma 3.

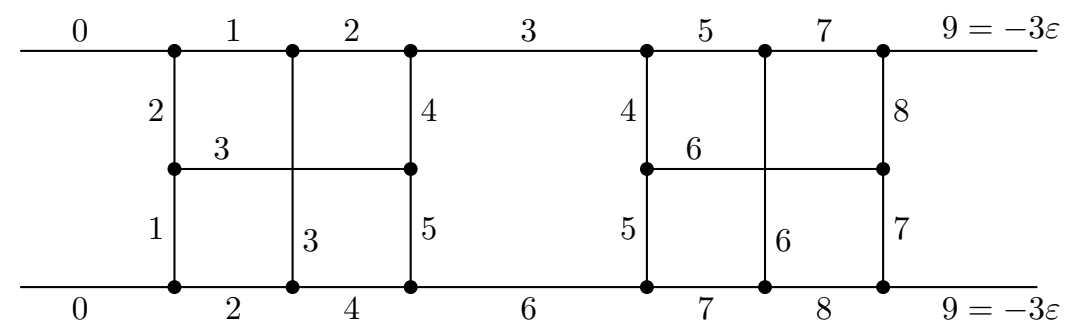

Figure 4: The colouring of two consecutive $B$-blocks of $B_{n}^{2}$ used in the proof of Lemma 3 . All colours are negated for better readability.

Lemma 4. If $\chi_{c}^{\prime}(G)=\frac{p}{q}$, where $p$ and $q$ are relatively prime positive integers, then $p \leqslant|E(G)|$ and $q \leqslant \alpha^{\prime}(G) \leqslant \frac{1}{2}|V(G)|$.

Lemma 5. Let $r=3+\varepsilon$ for some $0<\varepsilon<1$, and let $c$ be an edge $r$-colouring of a cubic graph $G$. If e, $e^{\prime} \in E(G)$ are at distance $d$ in the line graph $L(G)$, then $c\left(e^{\prime}\right) \in$ $[c(e)+t, c(e)+t+d \varepsilon]_{r}$ for some integer $d \leqslant t \leqslant 2 d$.

To prove the lower bounds, we need to study edge $(3+\varepsilon)$-colourings of the blocks $B$ and $A_{2}$. The following is proved by Mazák [6].

Lemma 6. [6] Let $0<\varepsilon<\frac{1}{4}, r=3+\varepsilon$, and $c$ be an edge $r$-colouring of $B$. Then $\left|c(a)-c\left(a^{\prime}\right)\right|_{r} \leqslant 2 \varepsilon$ and $\left|c(a)-c\left(a^{\prime}\right)\right|_{r}+\left|c(b)-c\left(b^{\prime}\right)\right|_{r} \leqslant 3 \varepsilon$.

If $c$ is an edge 3-colouring of $A_{2}$ such that $c(a)=c\left(a^{\prime}\right)$, then by the parity lemma, $c(b)=c\left(b^{\prime}\right)$ which is a contradiction since this gives an edge 3 -colouring of the Petersen graph. Thus by the parity lemma, in every edge 3-colouring of $A_{2}$, either $c(a)=c(b) \neq$ $c\left(a^{\prime}\right)=c\left(b^{\prime}\right)$ or $c(a)=c\left(b^{\prime}\right) \neq c\left(a^{\prime}\right)=c(b)$. In our next lemma, we prove an analogue of this observation for edge $(3+\varepsilon)$-colourings of $A_{2}$.

Lemma 7. Let $0<\varepsilon<\frac{1}{3}, r=3+\varepsilon$, and $c$ be an edge $r$-colouring of $A_{2}$. Then $\left|c(a)-c\left(a^{\prime}\right)\right|_{r}+\left|c(b)-c\left(b^{\prime}\right)\right|_{r} \geqslant 2-2 \varepsilon$.

Proof. Let $e_{0}$ be the unique edge of $A_{2}$ which is at distance 3 from $a, a^{\prime}, b, b^{\prime}$. We may assume that $c\left(e_{0}\right)=0$. Then by Lemma 5 , for every $e \in E\left(A_{2}\right), c(e) \in[t, t+d \varepsilon]_{r}$ where 
$d$ and $t$ are integers satisfying $1 \leqslant d \leqslant 3$ and $d \leqslant t \leqslant 2 d$. Since $\varepsilon<\frac{1}{3}$, each of these intervals has length strictly less than 1 , and thus it contains exactly one of the integers $0,1,2$. Let $\sigma(e) \in\{0,1,2\}$ be the integer corresponding to (the interval containing) $c(e)$. Since $c$ is an edge $r$-colouring, the mapping $\sigma$ is a proper edge 3 -colouring of $A_{2}$ and by the parity lemma, either $\sigma(a)=\sigma(b) \neq \sigma\left(a^{\prime}\right)=\sigma\left(b^{\prime}\right)$ or $\sigma(a)=\sigma\left(b^{\prime}\right) \neq \sigma\left(a^{\prime}\right)=\sigma(b)$. By symmetries of $A_{2}$, we may assume that the former holds. Now up to symmetry we either have

$$
\begin{aligned}
& \sigma(a)=\sigma(b)=0 \quad \text { and } \quad \sigma\left(a^{\prime}\right)=\sigma\left(b^{\prime}\right)=1, \quad \text { or } \\
& \sigma(a)=\sigma(b)=1 \quad \text { and } \quad \sigma\left(a^{\prime}\right)=\sigma\left(b^{\prime}\right)=2 .
\end{aligned}
$$

If we reduce the colours in $[-2 \varepsilon, 0]_{r}$ modulo $r$ to be in the real interval $[-2 \varepsilon, 0]$, then by (2) we have

$$
\left|c(a)-c\left(a^{\prime}\right)\right|_{r}=c\left(a^{\prime}\right)-c(a) \quad \text { and } \quad\left|c(b)-c\left(b^{\prime}\right)\right|_{r}=c\left(b^{\prime}\right)-c(b) .
$$

On the other hand, since $a^{\prime}$ and $b^{\prime}$ are at distance 3 from $b$ and $a$ respectively, there exist integers $3 \leqslant s, t \leqslant 6$ such that $c\left(a^{\prime}\right)-c(b) \in[s, s+3 \varepsilon]_{r}$ and $c\left(b^{\prime}\right)-c(a) \in[t, t+3 \varepsilon]_{r}$. Since $\sigma\left(a^{\prime}\right)-\sigma(b)=\sigma\left(b^{\prime}\right)-\sigma(a)=1$, we have $s=r=4$. Therefore $c\left(a^{\prime}\right)-c(b)$ and $c\left(b^{\prime}\right)-c(a)$ are both in the real interval $[1-\varepsilon, 1+2 \varepsilon]$ and we have

$$
\begin{aligned}
\left|c(a)-c\left(a^{\prime}\right)\right|_{r}+\left|c(b)-c\left(b^{\prime}\right)\right|_{r} & =c\left(a^{\prime}\right)-c(a)+c\left(b^{\prime}\right)-c(b) \\
& =c\left(a^{\prime}\right)-c(b)+c\left(b^{\prime}\right)-c(a) \geqslant 2(1-\varepsilon) .
\end{aligned}
$$

The above two lemmas give a lower bound on the circular chromatic index of type 2 generalized Blanuša snarks.

Corollary 8. For all $n \geqslant 2, \chi_{c}^{\prime}\left(B_{n+1}^{2}\right) \geqslant 3+\frac{2}{3 n+2}$.

Proof. The graph $B_{n+1}^{2}$ has $n$ copies of $B$ joined sequentially. Given an edge $(3+\varepsilon)-$ colouring of this graph, the difference between the colours of the semiedges $a^{\prime}$ and $b^{\prime}$ of the first copy of $B$, and the semiedges $a$ and $b$ of the last copy of $B$ is at most $n(3 \varepsilon)$ by Lemma 6 . On the other hand since these semiedges are joined to the semiedges of the copy of $A_{2}$, by Lemma 7 we have

$$
3 n \varepsilon \geqslant\left|c(a)-c\left(a^{\prime}\right)\right|_{r}+\left|c(b)-c\left(b^{\prime}\right)\right|_{r} \geqslant 2-2 \varepsilon .
$$

Solving for $\varepsilon$ we get $\varepsilon \geqslant \frac{2}{3 n+2}$.

For even $n \geqslant 2$, Theorem 2 follows by Lemma 3 and Corollary 8 . For odd $n$, the key is to prove that the inequality in the lower bound of Corollary 8 is strict. Before we present a proof of this fact, we need the following equivalent definition of circular edge colouring. Let $p, q$ be positive integers with $p / q \geqslant 2$. An edge $(p, q)$-colouring of a graph $G$ is any mapping $c: E(G) \rightarrow\{0,1, \ldots, p-1\}$, such that for any two adjacent edges $e$ and $e^{\prime}$ we have

$$
q \leqslant\left|c(e)-c\left(e^{\prime}\right)\right| \leqslant p-q .
$$

Note that $e \rightarrow c(e) / q$ defines an edge $p / q$-colouring of $G$. It is well-known that for every such $p, q$, a graph $G$ has an edge $p / q$-colouring if and only if it has an edge $(p, q)$-colouring. 
Lemma 9. For all odd $n \geqslant 3, \chi_{c}^{\prime}\left(B_{n+1}^{2}\right)>3+\frac{2}{3 n+2}$.

Proof. Let $G=B_{n+1}^{2}$. Suppose $\chi_{c}^{\prime}(G)=3+\frac{2}{3 n+2}$ and let $\psi$ be an edge $(9 n+8,3 n+2)-$ colouring of $G$. Let $\varepsilon=\frac{2}{3 n+2}, r=3+\varepsilon$, and $c$ be the edge $r$-colouring of $G$ defined by $c(e)=\psi(e) /(3 n+2)$. Note that for all $e \in E(G), c(e)$ is an integer multiple of $\varepsilon / 2$. Also note that since $n \geqslant 3, \varepsilon \leqslant \frac{2}{11}$. To clarify the notation, we denote $\varepsilon / 2$ by $\mu$ throughout this proof. Let $a_{i}$ and $b_{i}$ be the edges of $G$ corresponding to the semiedges $a$ and $b$ of the $i$ th copy of $B$ in $G$. Then $a_{i+1}$ and $b_{i+1}$ correspond to the semiedges $a^{\prime}$ and $b^{\prime}$ of the $i$ th copy of $B$ in $G$, and $a_{n+1}, b_{n+1}, a_{1}, b_{1}$ correspond respectively to the semiedges $a, b, a^{\prime}, b^{\prime}$ of the copy of $A_{2}$ in $G$.

Note that since $\chi_{c}^{\prime}(G)$ equals the lower bound of Corollary 8, all the inequalities in the proof of that lower bound are tight. Namely, $\left|c\left(a_{i+1}\right)-c\left(a_{i}\right)\right|_{r}+\left|c\left(b_{i+1}\right)-c\left(b_{i}\right)\right|_{r}=3 \varepsilon$ for all $1 \leqslant i \leqslant n$, and $\left|c\left(a_{n+1}\right)-c\left(a_{1}\right)\right|_{r}+\left|c\left(b_{n+1}\right)-c\left(b_{1}\right)\right|_{r}=2-2 \varepsilon=n(3 \varepsilon)$. Therefore by Lemma 6 , we may assume that for all $1 \leqslant i \leqslant n$,

$$
a_{i+1} \in\left[a_{i}+\varepsilon, a_{i}+2 \varepsilon\right]_{r} \text { and } b_{i+1} \in\left[b_{i}+\varepsilon, b_{i}+2 \varepsilon\right]_{r} .
$$

Since $a_{i}$ and $b_{i}$ are at distance 3 , by Lemma 5 we have $\left|c\left(a_{i}\right)-c\left(b_{i}\right)\right|_{r} \in\left[t_{i}, t_{i}+3 \varepsilon\right]_{r}$ for some integer $3 \leqslant t_{i} \leqslant 6$. Each of these intervals contains exactly one of the integers 0,1,2 and intervals corresponding to different numbers are disjoint. Let $\sigma_{i}$ be the unique member of $\left[t_{i}, t_{i}+3 \varepsilon\right]_{r} \cap\{0,1,2\}$. Since $|x|_{r} \leqslant r / 2$ for all $x, \sigma_{i} \neq 2$. Now by (3), $a_{i+1}-b_{i+1} \in\left[a_{i}-b_{i}-\varepsilon, a_{i}-b_{i}+\varepsilon\right]_{r}$. Thus $\sigma_{i+1}=\sigma_{i}$. Let $\sigma$ be the common value of all $\sigma_{i}$. It is easy to see that $\sigma=1$ is not compatible with either of the constraints (2). Therefore $\sigma=0$.

Consider the $i$ th $B$-block of $G$. Since $\left|c\left(a_{i+1}\right)-c\left(a_{i}\right)\right|_{r}+\left|c\left(b_{i+1}\right)-c\left(b_{i}\right)\right|_{r}=3 \varepsilon=6 \mu$, we may assume that $\left|c\left(a_{i+1}\right)-c\left(a_{i}\right)\right|_{r} \geqslant 3 \mu$. We also assume that $c(a)=0$. Thus $c\left(a_{i+1}\right) \in\{3 \mu, 4 \mu\}$ since it is an integer multiple of $\mu$.

Case 1. Let $c\left(a_{i+1}\right)=3 \mu$. Since $b_{i}$ and $b_{i+1}$ are both at distance 3 from $a_{i}$ and $a_{i+1}$, and since $\sigma=0$,

$$
\begin{aligned}
c\left(b_{i}\right), c\left(b_{i+1}\right) & \in\left[c\left(a_{i}\right)-2 \varepsilon, c\left(a_{i}\right)+2 \varepsilon\right]_{r} \cap\left[c\left(a_{i+1}\right)-2 \varepsilon, c\left(a_{i+1}\right)+2 \varepsilon\right]_{r} \\
& =[-4 \mu, 4 \mu]_{r} \cap[-\mu, 9 \mu]_{r} \\
& =[-\mu, 4 \mu]_{r}
\end{aligned}
$$

On the other hand, since one of the edges adjacent with $a_{i}$ has a colour in $[2,2+\varepsilon]_{r}$, at least one of the colours $c\left(b_{i}\right), c\left(b_{i+1}\right)$, is in $[6,6+3 \varepsilon]_{r}=[-4 \mu, 2 \mu]$. Thus the only possible way to get $\left|c\left(b_{i+1}\right)-c\left(b_{i}\right)\right|_{r}=3 \mu$ is that $c\left(b_{i}\right)=-\mu$ and $c\left(b_{i+1}\right)=2 \mu$.

Case 2. If $c\left(a_{i+1}\right)=4 \mu$, a similar argument shows that either $c\left(b_{i}\right)=0$ and $c\left(b_{i+1}\right)=$ $2 \mu$, or $c\left(b_{i}\right)=2 \mu$ and $c\left(b_{i+1}\right)=4 \mu$.

Note that in Case 1, $\left|c\left(a_{i}\right)-c\left(b_{i}\right)\right|_{r}=\left|c\left(a_{i+1}\right)-c\left(b_{i+1}\right)\right|_{r}=\mu$ while in Case 2, one of $\left|c\left(a_{i}\right)-c\left(b_{i}\right)\right|_{r}$ and $\left|c\left(a_{i+1}\right)-c\left(b_{i+1}\right)\right|_{r}$ is 0 while the other is $\varepsilon$. Therefore, these two colourings are not compatible with each other for consecutive copies of $B$, and the same case holds for all copies of $B$. Up to symmetries, in the first case we have $c\left(a_{1}\right)=0$, $c\left(b_{1}\right)=\mu, c\left(a_{n+1}\right)=1-2 \mu$, and $c\left(b_{n+1}\right)=1-\mu$, while in the second case we have 
$c\left(a_{1}\right)=0, c\left(b_{1}\right)=0, c\left(a_{n+1}\right)=1-3 \mu$, and $c\left(b_{n+1}\right)=1-\mu$. In either case we have $c\left(a_{n+1}\right)=c\left(b_{1}\right)+1-3 \mu$. On the other hand since $a_{n+1}$ is at distance 3 from $b_{1}$, by Lemma 5 we must have

$$
1-\frac{3 \varepsilon}{2}=c\left(a_{n+1}\right)-c\left(b_{1}\right) \in[t, t+3 \varepsilon]_{r},
$$

for some $3 \leqslant t \leqslant 6$. This contradicts the choice of $\varepsilon$.

The following lemma is the last ingredient we need in our proof of Theorem 2.

Lemma 10. Given an integer $n \geqslant 5$, there exist no positive integers $p \leqslant 12 n+15$ and $q \leqslant 4 n+5$ such that

$$
3+\frac{2}{3 n+2}<\frac{p}{q}<3+\frac{2}{3 n+1} .
$$

Proof. Suppose there exist $p$ and $q$ with the desired properties. Then

$$
p-3 q<\frac{2 q}{3 n+1} \leqslant \frac{8 n+10}{3 n+1} \leqslant \frac{25}{8},
$$

where the last inequality holds since $n \geqslant 5$. Therefore, $p-3 q \in\{1,2,3\}$. On the other hand, by (4) we have

$$
(p-3 q) \frac{3 n+1}{2}<q<(p-3 q) \frac{3 n+2}{2}
$$

This gives $(3 n+1) / 2<q<(3 n+2) / 2$ if $p-3 q=1$, and $3 n+1<q<3 n+2$ if $p-3 q=2$. These both contradict the fact that $q$ is an integer. If $p-3 q=3$, we similarly get $q \geqslant(9 n+4) / 2$ which implies $27 n+18 \leqslant 24 n+30$ since $p \leqslant 12 n+15$. This contradicts $n \geqslant 5$.

Note that $B_{n+1}^{2}$ has order $8 n+10$ and size $12 n+15$. Thus by Lemma 4 , if $\chi_{c}^{\prime}\left(B_{n+1}^{2}\right)=$ $p / q$ where $p$ and $q$ are relatively prime positive integers, then $p \leqslant 12 n+15$ and $q \leqslant 4 n+5$. Therefore for odd $n \geqslant 5$, Theorem 2 follows by Lemmas 3, 9, and 10 .

\section{$5 \quad$ Two Remaining Graphs}

For $n=1,3$, we had to use computers to settle the circular chromatic index of $B_{n+1}^{2}$. The main theoretical tool in such computer experiments is the concept of a tight cycle. Given an edge $r$-colouring $c$ of a graph $G$, we construct a digraph $H$ with $V(H)=E(G)$ in which there is an edge from $e$ to $e^{\prime}$ if $c\left(e^{\prime}\right)=c(e)+1$ modulo $r$. Any directed cycle in $H$ is called a tight cycle of $c$ (or of $G$ with respect to $c$ ). It is known (cf. [9] for example), that $\chi_{c}^{\prime}(G)=r$ if and only if every edge $r$-colouring of $G$ has a tight cycle.

For the second Blanuša snark $B_{2}^{2}$, we investigated all its edge $(7,2)$-colourings using a computer program and verified that they all have tight cycles. Thus $\chi_{c}^{\prime}\left(B_{2}^{2}\right)=7 / 2$. 
For $B_{4}^{2}$, Lemma 4 and the bounds proved in this paper give

$$
\chi_{c}^{\prime}\left(B_{4}^{2}\right) \in\left\{\frac{51}{16}, \frac{16}{5}\right\} .
$$

Using a computer program we verified that no Hamilton cycle of the line graph $L\left(B_{4}^{2}\right)$ can serve as a tight cycle in an edge $(51,16)$-colouring of $B_{4}^{2}$, thus proving $\chi_{c}^{\prime}\left(B_{4}^{2}\right)=16 / 5$.

The computer programs used to obtain the results of this section are available at the author's personal web page http://www.cecm.sfu.ca/ ${ }^{\sim m g h e b l e h / p r o g . ~}$

\section{References}

[1] P. Afshani, M. Ghandehari, M. Ghandehari, H. Hatami, R. Tusserkani, AND X. ZHU, Circular chromatic index of graphs of maximum degree 3, J. Graph Theory, 49 (2005), pp. 325-335.

[2] M. GHeBleh, The circular chromatic index of Goldberg snarks, Discrete Math., 307 (2007), pp. 3220-3225.

[3] M. Ghebleh, D. Král', S. Norine, and R. Thomas, The circular chromatic index of flower snarks, Electron. J. Combin., 13 (2006), pp. Note 20, 7 pp. (electronic).

[4] R. IsAACS, Infinite families of nontrivial trivalent graphs which are not Tait colorable, Amer. Math. Monthly, 82 (1975), pp. 221-239.

[5] T. Kaiser, D. KráL, And R. Škrekovski, A revival of the girth conjecture, J. Combin. Theory Ser. B, 92 (2004), pp. 41-53.

[6] J. MazÁK, Circular chromatic index of snarks, Master's thesis, Comenius University in Bratislava, 2007.

[7] J. J. Watkins, Snarks, in Graph theory and its applications: East and West (Jinan, 1986), vol. 576 of Ann. New York Acad. Sci., New York Acad. Sci., New York, 1989, pp. 606-622.

[8] D. B. West And X. Zhu, Circular chromatic index of Cartesian products of graphs, J. Graph Theory, (to appear).

[9] X. ZHu, Circular chromatic number: a survey, Discrete Math., 229 (2001), pp. 371410. 